\title{
Salvage Treatment with Cefiderocol Regimens in Two Intravascular Foreign Body Infections by MDR Gram- Negative Pathogens, Involving Non-Removable Devices
}

\author{
Marta Bodro · Marta Hernández-Meneses · Juan Ambrosioni (1) - Laura Linares • \\ Asunción Moreno · Elena Sandoval · Pol Olivas · María Hernández-Tejero · \\ José M. Miró · Francesc Marco $\cdot$ Alex Soriano
}

Accepted: December 9, 2020 / Published online: January 8, 2021

(c) The Author(s) 2021

\section{ABSTRACT}

Introduction: The objective of this study was to describe two challenging cases of intravascular foreign body infections caused by multidrugresistant Gram-negative pathogens requiring complex antimicrobial regimens including cefiderocol and successfully treated without implant removal.

Methods: Clinical charts and microbiological reports of the clinical cases.

M. Bodro · M. Hernández-Meneses · J. Ambrosioni $(\bowtie) \cdot$ L. Linares · A. Moreno · J. M. Miró ·

A. Soriano

Department of Infectious Diseases, Hospital ClínicIDIBAPS, University of Barcelona, Barcelona, Spain e-mail: jambrosioni@intramed.net

E. Sandoval

Department of Cardiovascular Surgery, Hospital Clínic-IDIBAPS, University of Barcelona, Barcelona, Spain

P. Olivas · M. Hernández-Tejero

Department of Hepatology. Hospital Clínic-

IDIBAPS, University of Barcelona, Barcelona, Spain

F. Marco

Microbiology Laboratory, CDB, ISGlobal, Hospital Clínic-IDIBAPS, University of Barcelona, Barcelona, Spain
Results: Case 1 included a left ventricular assist device (HEARTMATE $3^{\text {TM }}$ Abbot $^{\circledR}$ ) infection due to Achromobacter xylosoxidans, while case 2 included a portal prosthesis infection due to Pseudomonas aeruginosa. As the pathogens were multidrug-resistant (MDR), both cases required antimicrobial regimens with cefiderocol; treatment was successful without implant removal. Importantly, case 1 presented a probable, druginduced thrombocytopenia, a non-previously described side effect related to cefiderocol.

Conclusion: Cefiderocol may be an additional, promising drug to the available arsenal, even for challenging foreign body infections caused by MDR Gram-negative pathogens.

Keywords: Cefiderocol; Foreign body infections; Gram-negative bacteria; Multidrug resistance 


\section{Key Summary Points}

Multidrug-resistant (MDR) Gram-negative (GN) infections have become a major health problem worldwide.

Intravascular infections are particularly challenging when they involve nonremovable devices and are caused by MDR microorganisms.

Cefiderocol is a promising added drug to the available armamentaria against MDR Gram-negative bacteria.

The cases reported here suggest the potential use of cefiderocol in foreignbody infections.

Cefiderocol could induce drug-related thrombocytopenia.

\section{DIGITAL FEATURES}

This article is published with digital features, including a summary slide, to facilitate understanding of the article. To view digital features for this article go to https://doi.org/10.6084/ m9.figshare.13348340.

\section{INTRODUCTION}

Multidrug-resistant (MDR) Gram-negative infections have become a major health problem worldwide [1]. Such infections are associated with high morbidity and mortality, affecting patients with poor medical conditions who more often receive inadequate empirical therapy and whose final treatment, in most cases, includes nephrotoxic antibiotics such as amikacin or colistin [2].

In Gram-negative bacteria, the bacterial outer membrane (absent in Gram-positive bacteria) can restrict $\beta$-lactam entry and contribute to a concentration of $\beta$-lactamase molecules. High levels of resistance can occur if either reduced entry or increased efflux sufficiently excludes $\beta$-lactam molecules from the periplasmic space, or a heavy concentration of $\beta$-lactamase molecules is present. To increase the efficacy of well-known antibiotics, clinicians can use the "Trojan horse" strategy, in which drugs are transported into the periplasmic space via the iron uptake pathway. That stated, cefiderocol is a cephalosporin with a catechol group in the side chain at position 3 that binds free iron (siderophore) and confers additional stability against a wide range of serine and metallo ß-lactamases[3, 4]. In recent surveillance studies that tested bacterial clinical isolates from the United States, Asia-Pacific and Europe, $\mathrm{MIC}_{90}$ for cefiderocol against meropenem-non-susceptible (MIC $>4 \mathrm{mg} / \mathrm{L}$ ) isolates of Enterobacteriaceae, Pseudomonas aeruginosa (Pae), Acinetobacter baumannii and Stenotrophomonas maltophilia [5] was $\leq 4 \mathrm{mg} / \mathrm{L}$ in all cases, except for Enterobacteriaceae harboring SHV/TEM extended-spectrum ß-lactamases (ESBL), NDM and KPC-2 in the same strain that have MIC values of $0.5-8 \mathrm{mg} / \mathrm{L}$ [6-9].

In vivo efficacy of cefiderocol has been documented in multiple, humanized drug exposure murine and rat models of infection using different MDR and extremely drug-resistant (XDR) strains [10]. In humans, efficacy and safety of this cefalosporin $(2 \mathrm{~g} / 8 \mathrm{~h})$ was evaluated in a randomized clinical trial against imipenem/cilastatin $(1 \mathrm{~g} / 8 \mathrm{~h})$, with both being administered intravenously for 7-14 days for $1 \mathrm{~h}$ in hospitalized adults with urinary tract infections [11] and in patients with nosocomial pneumonia[12]. However, more reports assessing the use of cefiderocol are necessary to understand the efficacy and safety of this drug.

We describe two severe infections caused by MDR-Achromobacter xylosoxidans (Ax) and XDR$P a e$, in which both involved non-removable intravascular devices and treatment was successful with combined regimens with cefiderocol.

\section{METHODS}

We described the characteristics and outcome of two patients who received cefiderocol for severe 


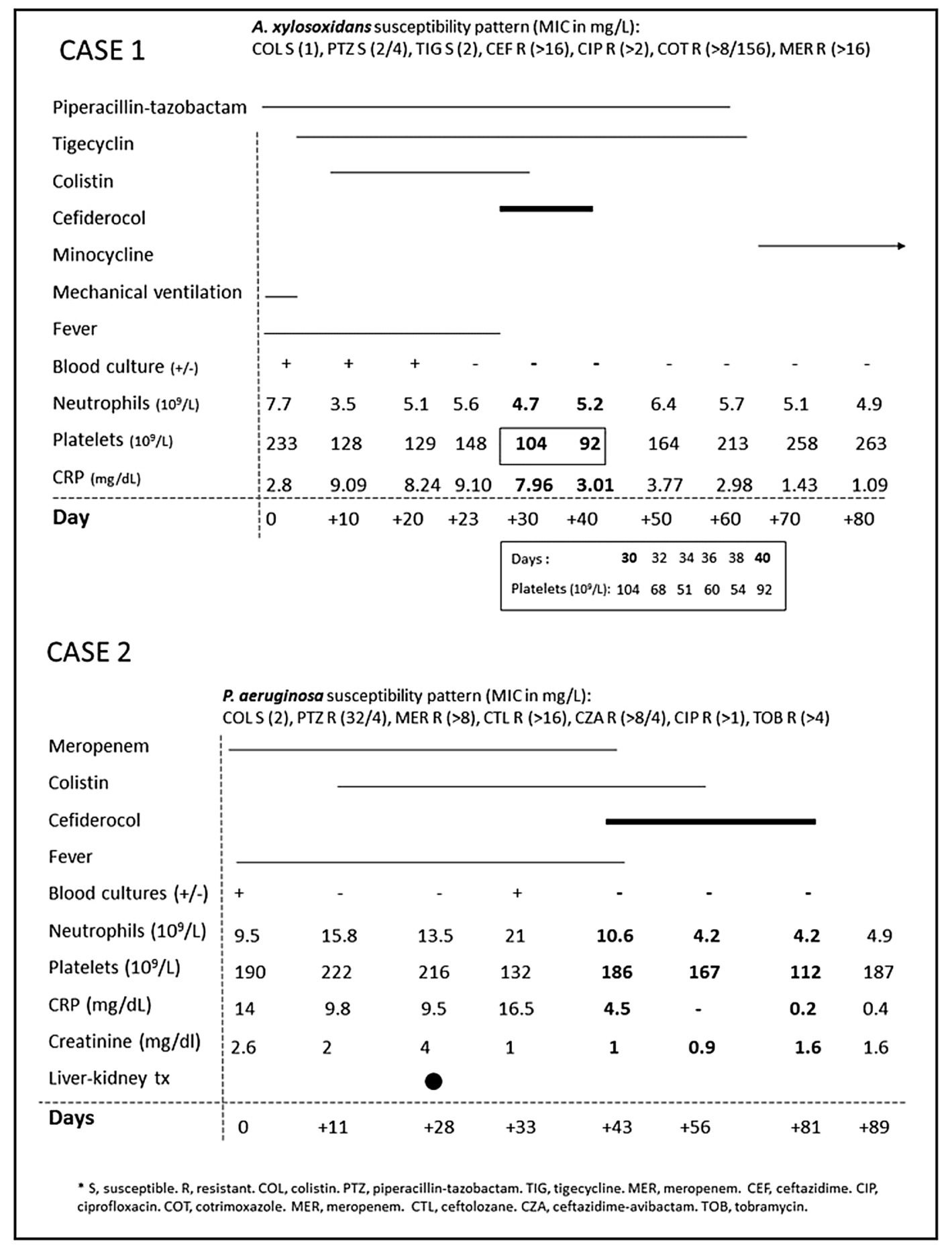

Fig. 1 Descriptions of pathogen susceptibility patterns, antibiotic consumption, blood culture results and main clinical and biochemical parameters in case $1(\mathbf{a})$ and $2(\mathbf{b})$ 
infections caused by MDR pathogens. We also reviewed published data of randomized trials, case reports and in vitro activity of cefiderocol. Written informed consent for publication of this article was obtained from both patients. Patients were identified by reviewing records, no formal evaluation of ethics committee was required.

\section{RESULTS}

\section{Patient 1}

A 66-year-old man with refractory chronic heart failure with poor response to medical treatment, and a slow-growing lung adenocarcinoma contraindicating a heart transplant, received a left ventricular assist device (HEARTMATE $3^{\mathrm{TM}}$ Abbot). While under mechanical ventilation, he presented with a purulent tracheobronchitis due to MDR- $A x$. The patient received piperacillin-tazobactam at $4.5 \mathrm{~g} / 6 \mathrm{~h}$ in accordance with the susceptibility pattern (Fig. 1a); however, at day 5, the patient remained febrile and blood cultures tested positive for the same strain. The patient then received tigecycline $100 \mathrm{mg} / 12 \mathrm{~h}$ and colistin 3 $\mathrm{MU} / 8 \mathrm{~h}$ intravenously. Bacteriemia persisted for 21 days. A cardiac and corporal ${ }^{18}$ F-FDG-positron emission tomography, combined with computed tomography, did not show septic metastases. Although the device did not reveal any relevant uptake, long-lasting bacteremia and the absence of other identified active foci led to the assumption of a device-associated infection. Cefiderocol was reported as sensitive in our laboratory, with an inhibition halo of $21 \mathrm{~mm}$. The drug was therefore requested through the compassionate use program and started 25 days after the first positive blood culture. Yet, a final blood culture performed just prior to cefiderocol administration tested negative. The administered dose was $2 \mathrm{~g} / 8 \mathrm{~h}$ in a $3-\mathrm{h}$ infusion, while piperacillin-tazobactam and tigecycline remained. Colistin was discontinued due to renal function deterioration. Weekly surveillance blood cultures tested negative during treatment. Within 3 days of cefiderocol administration, C-reactive protein serum concentration progressively decreased and patient's health improved. Within 14 days of dual B-lactam therapy, the patient developed thrombocytopenia $\left(53,000\right.$ platelets $\left./ \mathrm{mm}^{3}\right)$. Cefiderocol was, therefore, discontinued while piperacillin-tazobactam and tigecycline were continued for 28 additional days. Platelet count returned to baseline levels within 3 days of cefiderocol discontinuation. Oral suppressive therapy with minocycline was prescribed; at 12-month follow-up, the patient was in good clinical condition and blood cultures repeatedly tested negative.

\section{Patient 2}

A 55-year-old man was admitted to the hospital for portal revascularization. He presented with a thrombophilic disorder that caused portal cavernomatosis and acute mesenteric thrombosis that required ileal resection. Additionally, the patient had underwent a renal transplant in 2013.

Portal revascularization was achieved via a transjugular intrahepatic portosystemic shunt insertion; however, complications arose due to a bilioportal fistula and bacteremia caused by XDR-Pae. The patient, therefore, received meropenem $2 \mathrm{~g} / 8 \mathrm{~h}$ as well as colistin $3 \mathrm{MU} / 8 \mathrm{~h}$ due to persistent fever. Infection persisted for more than 1 month, suggesting a shunt infection; the patient developed kidney function deterioration (Fig. 1b). The patient then underwent a combined kidney-liver transplant, although the distal part of the portal prosthesis (approximately 20\%) could not be removed during surgery. In spite of intravenous colistin being administered before and after transplant surgery, the patient presented with bacteremia due to the same XDR-Pae, presumably from the residual shunt, within 5 days of surgery. The bacteremia probably superinfected an intraabdominal hematoma, subsequently requiring surgical drainage. XDR-Pae grew on both the surgical cultures of the shunt and the intra-abdominal hematoma. Disk diffusion test for cefiderocol showed an inhibition zone of $23 \mathrm{~mm}$, and it was therefore requested through a compassionate use program. The patient 
initially received intravenous cefiderocol $2 \mathrm{~g} /$ $8 \mathrm{~h}$ in a 3-h infusion and colistin $3 \mathrm{MU} / 8 \mathrm{~h}$ for 2 weeks, with an additional 4 weeks of cefiderocol as monotherapy. No adverse events were observed during antibiotic treatment. The patient's evolution with respect to persistent bacteremia by XDR-Pae was good, with negative blood cultures, no further episodes of fever, and infection-free status within 3 months of antibiotic treatment discontinuation.

Written informed consent for inclusion in this report was obtained from both patients.

\section{DISCUSSION}

Our report is the first to describe the outcome of two patients with severe infections due to Gram-negative bacteria that involved non-removable intravascular devices. In both cases, treatment with combined regimens with cefiderocol was successful and no implant removal was necessary. A recent study using a worldwide collection of 8954 clinical isolates of Pae, Enterobacteriaceae, Acinetobacter baumannii, Stenotrophomonas maltophilia and Burkholderia cepacia, collected from 2015 to 2016, revealed that cefiderocol demonstrated potent in vitro activity $(\mathrm{MIC} \leq 4 \mathrm{mg} / \mathrm{L})$ against the majority $(99.4 \%)$ of these isolates, including MDR strains $[8,13]$.

We assumed that both patients presented with intravascular device-associated infection, given bacteremia persistence and in spite of correct antibiotic administration per in vitrotested susceptibilities and no other secondary foci. Cefiderocol may have had a role in both cases. In the first case, the role of cefiderocol may be less relevant, as blood cultures tested negative upon drug initiation. However, there was a clinical and analytical improvement, such as the rapid CRP decrease after administration of the drug. As this patient continues receiving suppressive therapy with oral minocycline $100 \mathrm{mg} / 12 \mathrm{~h}$, it is not possible to confirm a complete cure of the infection. However, the commonly reached serum concentration of minocycline is $<2 \mathrm{mg} / \mathrm{L}$ and the MIC of this drug for this strain was $3 \mathrm{mg} / \mathrm{L}$. Its activity may have therefore been only partial, which makes it difficult to assume that minocycline alone is controlling the infection.

Cefiderocol has also been reported in recent cases of intravascular devices/infections. Trecarichi et al. reported a case of XDR-A. baumannii-causing pneumonia and bloodstream infection in a patient on extracorporeal membrane oxygenation[14]. Furthermore, a separate, recent report of endocarditis due to XDR-Pae was successfully treated with cefiderocol[15]. Although the patient underwent aortic valve surgery after six doses of cefiderocol, the valve culture was negative. Thus, cefiderocol may have potential activity against biofilm-forming Gram-negative pathogens, which are eager to capture iron. Infected devices should be removed whenever possible. When removal cannot be achieved, antimicrobials retaining anti-biofilm activity should be prioritized. A recent report suggests that cefiderocol may reduce biofilm and may inhibit planktonic growth of Gram-negative bacteria [16].

Patients in our first case and in that reported by Edgeworth et al. developed thrombocytopenia and neutropenia, respectively. Interestingly, both patients received cefiderocol and a second beta-lactam (piperacillin-tazobactam and meropenem, respectively) for $\geq 2$ weeks. Important side effects of this kind have never been described in the literature.

In conclusion, in challenging MDR and XDR Gram-negative infections, cefiderocol may be an additional, promising drug to the therapeutic options available for clinicians, even in cases of difficult-to-treat, intravascular foreign body infections with non-removable devices.

\section{ACKNOWLEDGEMENTS}

We acknowledge the Infectious Disease, Microbiology, Cardiology, Pharmacy Departments, as well as the Liver Transplant Unit for their contributions. Thank you Anthony Armenta for providing medical editing assistance for the report at hand. JMM received a personal 80:20 research grant from Institut d'Investigacions Biomèdiques August Pi i Sunyer (IDIBAPS), Barcelona, Spain, during 2017-21. 
Funding. No funding or sponsorship was received for this study or publication of this article.

Authorship. All named authors meet the international Committee of Medical Journal Editors criteria for authorship for this article, take responsibility for the integrity of the work as a whole, and have given their approval for this version to be published.

Authorship Contributions. $\mathrm{MB}$ and $\mathrm{MH}-\mathrm{M}$ have contributed equally.

Disclosures. Marta Bodro, Marta HernandezMeneses, Laura Linares, Asunción Moreno, Elena Sandoval, Pol Olivas, María HernándezTejero and Francesc Marco have nothing to disclose. José M. Miró received a personal 80:20 research grant from Institut d'Investigacions Biomèdiques August Pi i Sunyer (IDIBAPS), Barcelona, Spain, during 2017-21. Alex Soriano is an Editor-in-Chief of this journal. Juan Ambrosioni is an Editorial Board member of this journal.

Compliance with Ethics Guidelines. Written informed consent for publication of this article was obtained from both patients. Patients were identified by reviewing records, no formal evaluation of ethics committee was required.

Open Access. This article is licensed under a Creative Commons Attribution-NonCommercial 4.0 International License, which permits any non-commercial use, sharing, adaptation, distribution and reproduction in any medium or format, as long as you give appropriate credit to the original author(s) and the source, provide a link to the Creative Commons licence, and indicate if changes were made. The images or other third party material in this article are included in the article's Creative Commons licence, unless indicated otherwise in a credit line to the material. If material is not included in the article's Creative Commons licence and your intended use is not permitted by statutory regulation or exceeds the permitted use, you will need to obtain permission directly from the copyright holder. To view a copy of this licence, visit http://creativecommons.org/licenses/by$\mathrm{nc} / 4.0 /$.

\section{REFERENCES}

1. Logan LK, Weinstein RA. The epidemiology of carbapenem-resistant enterobacteriaceae: the impact and evolution of a global menace. J Infect Dis. 2017;215:S28-36.

2. Ordooei Javan A, Shokouhi S, Sahraei Z. A review on colistin nephrotoxicity. Eur J Clin Pharmacol. 2015;71:801-10.

3. Ito A, Nishikawa T, Matsumoto S, Yoshizawa $H$, Sato T, Nakamura R, et al. Siderophore cephalosporin cefiderocol utilizes ferric iron transporter systems for antibacterial activity against Pseudomonas aeruginosa. Antimicrob Agents Chemother. 2016;60:AAC.01405-16.

4. Zhanel GG, Golden AR, Zelenitsky S, Wiebe K, Lawrence CK, Adam HJ, et al. Cefiderocol: a siderophore cephalosporin with activity against carbapenem-resistant and multidrug-resistant gramnegative bacilli. Drugs [Internet]. 2019;79:271-89. https://doi.org/10.1007/s40265-019-1055-2.

5. Jacobs MR, Abdelhamed AM, Good CE, Rhoads DD, Hujer KM, Hujer AM, et al. ARGONAUT-I: activity of cefiderocol (S-649266), a siderophore cephalosporin, against gram-negative bacteria, including carbapenem-resistant nonfermenters and enterobacteriaceae with defined extended-spectrum $\beta$ lactamases and carbapenemases. Antimicrob Agents Chemother. 2018;63(1):e01801-18.

6. Falagas ME, Skalidis T, Vardakas KZ, Legakis NJ, Hellenic Cefiderocol Study Group. Activity of cefiderocol (S-649266) against carbapenem-resistant Gram-negative bacteria collected from inpatients in Greek hospitals. J Antimicrob Chemother. 2017;72:1704-8.

7. Yamano Y. In vitro activity of cefiderocol against a broad range of clinically important gram-negative bacteria. Clin Infect Dis. 2019;69(Suppl 7):S544-51.

8. Karlowsky JA, Hackel MA, Tsuji M, Yamano Y, Echols R, Sahm DF. In Vitro Activity Of Cefiderocol, A Siderophore Cephalosporin, Against Gram-Negative Bacilli Isolated By Clinical Laboratories in North America and Europe in 2015-2016: SIDEROWT-2015. Int J Antimicrob Agents. 2019;53:456-66.

9. Hackel MA, Tsuji M, Yamano Y, Echols R, Karlowsky JA, Sahm DF. In vitro activity of the 
siderophore cephalosporin, cefiderocol, against carbapenem-nonsusceptible and multidrug-resistant isolates of gram-negative bacilli collected worldwide in 2014 to 2016. Antimicrob Agents Chemother. 2018;62(2):e01968-17.

10. Monogue ML, Tsuji M, Yamano Y, Echols R, Nicolau DP. Efficacy of humanized exposures of cefiderocol (S-649266) against a diverse population of gram-negative bacteria in a murine thigh infection model. Antimicrob Agents Chemother. 2017;61(11):e01022-17.

11. Portsmouth $\mathrm{S}$, van Veenhuyzen $\mathrm{D}$, Echols $\mathrm{R}$, Machida M, Ferreira JCA, Ariyasu M, et al. Cefiderocol versus imipenem-cilastatin for the treatment of complicated urinary tract infections caused by Gram-negative uropathogens: a phase 2, randomised, double-blind, non-inferiority trial. Lancet Infect Dis. 2018;18:1319-28.

12. Wunderink RG, Matsunaga Y, Ariyasu M, Clevenbergh P, Echols R, Kaye KS, et al. Cefiderocol versus high-dose, extended-infusion meropenem for the treatment of Gram-negative nosocomial pneumonia (APEKS-NP): a randomised, double-blind, phase 3, non-inferiority trial. Lancet Infect Dis [Internet]. 2020 [cited 2020 Nov 23]; https://linkinghub. elsevier.com/retrieve/pii/S1473309920307313. Accessed 24 Nov 2020.

13. Kazmierczak KM, Tsuji $M$, Wise MG, Hackel $M$, Yamano $\mathrm{Y}$, Echols $\mathrm{R}$, et al. In vitro activity of cefiderocol, a siderophore cephalosporin, against a recent collection of clinically relevant carbapenemnon-susceptible Gram-negative bacilli, including serine carbapenemase- and metallo- $\beta$-lactamaseproducing isolates (SIDERO-WT-2014. Int $\mathrm{J}$ Antimicrob Agents. 2019;53:177-84.

14. Trecarichi EM, Quirino A, Scaglione V, Longhini F, Garofalo E, Bruni A, et al. Successful treatment with cefiderocol for compassionate use in a critically ill patient with XDR Acinetobacter baumannii and KPCproducing Klebsiella pneumoniae: a case report. J Antimicrob Chemother [Internet]. 2019 [cited 2019 Oct 25];74:3399-401. http://www.ncbi.nlm. nih.gov/pubmed/31369095. Accessed 24 Nov 2020.

15. Edgeworth JD, Merante D, Patel S, Young C, Jones $\mathrm{P}$, Vithlani $\mathrm{S}$, et al. Compassionate use of cefiderocol as adjunctive treatment of native aortic valve endocarditis due to extremely drug-resistant Pseudomonas aeruginosa. Clin Infect Dis [Internet]. 2019 [cited 2019 Jul 4];68:1932-4. https://academic.oup. com/cid/article/68/11/1932/5174241. Accessed 24 Nov 2020.

16. Pybus CA, Felder-Scott C, Obuekwe V, Greenberg DE. Cefiderocol retains anti-biofilm activity in multi-drug resistant gram-negative pathogens. Antimicrob Agents Chemother [Internet]. Antimicrob Agents Chemother; 2020 [cited 2020 Nov 24]; http://www.ncbi.nlm.nih.gov/pubmed/33199383. Accessed 24 Nov 2020. 